\title{
Preoperative Erythrocyte Aggregation Exponent May Affect Postoperative Risk of Deep Venous Thrombosis in Patients With Primary Knee Osteoarthritis
}

\section{Dai Xiaoyu}

The Third Affiliated Hospital of Soochow University

\section{Ying Pu}

Changshu Hospital, Nanjing University of Chinese Medicine

\section{Xu Peng}

The Third Affiliated Hospital of Soochow University

Huang Zhihui

The Third Affiliated Hospital of Soochow University

\section{Li Huan}

The Third Affiliated Hospital of Soochow University

\section{Zhang Naidong}

The Third Affiliated Hospital of Soochow University

\section{Ding Wenge ( $\nabla$ flying_ding@126.com )}

The Third Affiliated Hospital of Soochow University

\section{Research Article}

Keywords: Osteoarthritis, Knee, Erythrocyte aggregation, Total knee arthroplasty, Deep venous thrombosis

Posted Date: March 8th, 2021

DOI: https://doi.org/10.21203/rs.3.rs-284842/v1

License: (9) This work is licensed under a Creative Commons Attribution 4.0 International License. Read Full License 


\section{Abstract}

Background: The factors leading to venous thromboembolism (VTE) have been known for a long time. Previous data has suggested a potential link between erythrocyte aggregation (EA) and deep vein thrombosis (DVT). Considering a relatively large number of patients who need total knee arthroplasty (TKA) surgery due to primary knee osteoarthritis (KOA), this study is tried to verify whether preoperative EA may have an effect on postoperative DVT after TKA in these patients.

Methods: A total of 750 KOA patients accepted unilateral TKA were enrolled from March 2010 to May 2020. On the third day after surgery, they all accepted the color Doppler ultrasound examination of both legs for deep veins and were subsequently divided into DVT and non-DVT groups. We made a thorough analysis to demonstrate the specific association of EA exponent before TKA and DVT risk after TKA.

Results: Low EA exponent was a significant risk factor for DVT in patients with primary KOA $(p<0.05)$, especially in females after stratifying by gender $(p<0.05)$.

Conclusions: Our results suggested that low EA before TKA was substantially associated with a high post-surgery DVT risk in primary KOA patients. Timely and effective anticoagulant therapy for such patients was required.

\section{Introduction}

Osteoarthritis $(\mathrm{OA})$ is the most common degenerative joint disease and more prevalent in middle-aged and elderly people, which can cause joint pain, stiffness, and functional limitation[1, 2]. The fact is that there still exist no specific disease-modifying OA drugs and treatment is limited to palliation of pain and retention of joint function $[3,4]$. OA usually occurs in the knee joint and TKA is quite an important therapeutic method for terminal-stage KOA patients[5]. DVT is a critical manifestation of venous thromboembolism (VTE) and well recognized as one of the most severe complications after TKA, the incidence of which ranged from about $18.1-48.6 \%$ without thrombosis prevention in Asian patients[6]. A recent Nanjing Study reported that postoperative DVT occurred in $18.0 \%$ of the patients after total joint arthroplasty with low molecular weight heparin (LMWH) or Rivaroxaban for DVT prophylaxis[7]. It is welldescribed that DVT can lead to pulmonary embolism which is potentially fatal[8].

Epidemiological studies have confirmed a lot of hereditary and acquired DVT risk factors [9, 10], and some of them are related to hemorheological changes such as increased fibrinogen (FIB) levels and EA[11]. Notably, evidence strongly suggests erythrocytes are involved in venous thrombi by specific interactions and culminating in the formation of a erythrocyte- and fibrin-rich venous thrombus[12]. Two recent reviews explicitly indicated that both quantitative and qualitative changes in erythrocytes have biologically and clinically important functions in thrombogenesis and its disorders[13, 14]. Thereinto, erythrocytes hyper-aggregation is considered as a classic prothrombotic abnormality in first time and recurrent DVT patients[15]. Previously, Alt E et al. found that patients showed higher EA than controls in the acute phase of DVT. Yu FTH et al. also suggested that a remarkable elevation in EA promotes 
thrombosis in rabbit femoral veins, confirming a pathophysiological role in DVT onset[15]. Combined with the existing preventive antithrombotic guidelines after TKA and the relatively high incidence of KOA, the importance of targeted and timely anticoagulant therapies for KOA patients who need to undergo TKA should be noticeable. Identification of new risk factors associated with DVT can result in more comprehensive insights about prevention, diagnostic and treatment options for DVT. This study firstly aimed to further provide insight into a better understanding of DVT prevention for such patients by analyzing the potential correlation between preoperative EA and postoperative DVT.

\section{Materials And Methods}

\section{Patients.}

From March 2010 and May 2020, a total of 750 KOA patients (166 males and 604 females) who underwent TKA in our department were consecutively enrolled according to the exclusion criteria[16].

This study was approved by the ethical committee of our hospital and each patient needed to sign an informed consent form.

Weight and height were uniformly measured by trained nurses on the first day of admission and body mass index (BMI) was subsequently calculated. Some other clinical data including age, gender, and the history of diabetes, hypertension, malignancy, cardiovascular diseases (ie, diseases originate from atherothrombosis including myocardial infract, stable or unstable angina, or stoke), VTE events, steroids or estrogens use, smoking, surgery, and blood transfusion was collected according to patients' self-report and recorded.

\section{Blood sample testing and TKA}

A day after admission, three tubes of venous blood of each patient was sampled at 7 a.m. and measured in the clinical laboratory. One tube of blood used for testing EA was done by LBY-N6C automatic hemorheology instrument (Precil Co., CHN). In addition, parameters of coagulation function which consist of FIB, D-dimer, prothrombin time (PT), activated partial thromboplastin time (APTT), and thrombin time (TT) were examined with CA-7000 automatic blood coagulation analyzer (Sysmex Co., JPN). Platelet count and serum levels of triglycerides (TG), total cholesterol (TC), high density lipoprotein cholesterol (HDL-C), low density lipoprotein cholesterol (LDL-C), apolipoprotein A1 (apo A1), and apolipoprotein B (apo B) were evaluated by HITACHI 7600-20 fully biochemical auto-analyser (HITACHI, JPN).

All TKAs were performed under general anesthesia with the tourniquet. Surgical process of TKA consisted of condylar-type and posterior cruciate substituting cemented arthroplasty via a knee midline skin incision and a deep medial parapatellar approach. The average using time of tourniquet was 40 minutes during TKA. A drainage tube was placed in the joint for no more than 48 hours. Postoperatively, low molecular weight heparin (LMWH) was applied for DVT prevention every day for each patient and its firsttime use was started 10 hours after surgery. Patients were requested to do flexion and extension exercises of lower extremity joints and active muscles contraction at the early time after TKA. For each 
patient, some relevant symptoms and signs of acute DVT such as redundancy and tenderness in the calf were closely monitored.

\section{Diagnosis and management of DVT}

On the 3rd day postoperatively, ultrasound examination of bilateral lower extremity veins was done in each patient to determine the presence of DVT based on the results from Rabinov and Paulin[17]. The examiner was blinded to the intraoperative procedure and the preoperative hemorheological parameters. LMWH was used for DVT treatment and DVT patients continued with anticoagulation therapy even after discharge and the entire anticoagulant period should be at least three months.

\section{Statistical analysis}

SPSS software version 22.0 was used to do an analysis. The $\chi^{2}$ test was conducted to compare differences in categorical variables. Mean values were presented with their standard deviation (SD) and the Student's $t$ test was adopted to make a comparison. Preoperative confounding factors in this study were all adjusted for the multivariate logistic regression analysis. Still, a receiver operating characteristic (ROC) curve was made to evaluated the predictive value of the potential indicator. For all the tests, differences were considered significant when $p<0.05$.

\section{Results}

\section{Baseline characteristics}

In Table 1, there were 176 DVTs in KOA patients and the total incidence of postoperative DVT was $23.5 \%$ (176/750). Notably, female patients had a higher DVT incidence (25.1\%) than males (16.7\%). Through systematically analyzing and comparing, we could find that DVT patients had a higher TC $(p=0.010)$ and LDL-C levels $(p=0.018)$ and a lower smoking rate $(p=0.015)$ than non-DVT patients. In addition, no significant differences were observed in mean age and BMI, gender, diabetes, hypertension, malignancy, cardiovascular diseases, VTE events, steroids or estrogens use, surgery history, and blood transfusion history, blood levels of TG, HDL-C, apo A1, apo B, FIB, and D-dimer, platelet count, PT, APTT, and TT ( $p$ > 0.05). 
Table 1

Comparisons of general characteristics

\begin{tabular}{|c|c|c|c|}
\hline General characteristics & DVT patients & Non-DVT patients & $p$ value \\
\hline Patients, n & 176 & 574 & - \\
\hline Mean age, y (SD) & $67.18(7.435)$ & $66.62(8.185)$ & 0.416 \\
\hline Females, y (SD) & $67.04(7.678)$ & $65.86(8.393)$ & 0.126 \\
\hline Males, y (SD) & $68.08(5.710)$ & $69.50(6.624)$ & 0.330 \\
\hline Male gender (males/females, n) & $24 / 152$ & $120 / 454$ & $0.032^{\mathrm{a}}$ \\
\hline Mean BMI, kg/m² (SD) & $26.45(3.974)$ & $26.05(3.872)$ & 0.241 \\
\hline Females, $\mathrm{kg} / \mathrm{m}^{2}(\mathrm{SD})$ & $26.56(4.110)$ & $26.21(3.933)$ & 0.341 \\
\hline Males, $\mathrm{kg} / \mathrm{m}^{2}(\mathrm{SD})$ & $25.74(2.942)$ & $25.48(3.591)$ & 0.744 \\
\hline Diabetes mellitus (with/without, $n$ ) & $30 / 146$ & $86 / 488$ & $0.508^{\mathrm{a}}$ \\
\hline Hypertension (with/without, n) & $88 / 88$ & $290 / 284$ & $0.903^{a}$ \\
\hline Malignancy (with/without, $n$ ) & $8 / 168$ & $10 / 564$ & $0.065^{b}$ \\
\hline Cardiovascular events (with/without, n) & $12 / 164$ & $62 / 512$ & $0.121^{\mathrm{a}}$ \\
\hline VTE events (with/without, n) & $12 / 164$ & $44 / 530$ & $0.708^{\mathrm{a}}$ \\
\hline Steroids or estrogens use (with/without, $n$ ) & $8 / 168$ & $48 / 526$ & $0.092^{\mathrm{a}}$ \\
\hline Smoking (with/without, n) & $4 / 172$ & $42 / 532$ & $0.015^{a}$ \\
\hline Surgery history (with/without, n) & $34 / 142$ & $126 / 448$ & $0.456^{\mathrm{a}}$ \\
\hline Blood transfusion history (with/without, n) & $26 / 150$ & $86 / 488$ & $0.946^{\mathrm{a}}$ \\
\hline $\mathrm{TG}, \mathrm{mmol} / \mathrm{L}(\mathrm{SD})$ & $1.41(0.644)$ & $1.50(0.981)$ & 0.213 \\
\hline $\mathrm{TC}, \mathrm{mmol} / \mathrm{L}(\mathrm{SD})$ & $4.81(0.832)$ & $4.62(0.890)$ & 0.010 \\
\hline HDL-C, mmol/L (SD) & $1.35(0.342)$ & $1.31(0.330)$ & 0.096 \\
\hline
\end{tabular}

$n$ number, $y$ years, $B M /$ body mass index, $S D$ standard deviation, $V T E$ venous thrombus embolism, $T G$ triglycerides, $T C$ total cholesterol, $H D L-C$ high density lipoprotein cholesterol, $L D L-C$ low density lipoprotein cholesterol, apo $A 1$ apolipoprotein $A 1$, apo $B$ apolipoprotein, $F / B$ fibrinogen, $P T$ prothrombin time, $A P T T$ activated partial thromboplastin time, $T T$ thrombin time

a. Pearson Chi-square test

b. Continuous correction test 


\begin{tabular}{|c|c|c|c|}
\hline General characteristics & DVT patients & Non-DVT patients & $p$ value \\
\hline LDL-C, mmol/L (SD) & $2.57(0.665)$ & $2.44(0.625)$ & 0.018 \\
\hline Apo A1 & $1.24(0.211)$ & $1.21(0.219)$ & 0.086 \\
\hline Apo B & $0.95(0.207)$ & $0.93(0.213)$ & 0.322 \\
\hline Platelet count, $10^{12} / \mathrm{L}$ (SD) & $20311(61.265)$ & $209.16(71.910)$ & 0.314 \\
\hline $\mathrm{FIB}, \mathrm{g} / \mathrm{L}(\mathrm{SD})$ & $3.18(0.745)$ & $3.26(0.794)$ & 0.218 \\
\hline D-dimer, mg/L (SD) & $0.65(1.270)$ & $0.74(1.266)$ & 0.394 \\
\hline PT, s (SD) & $11.31(0.736)$ & $11.20(1.047)$ & 0.183 \\
\hline APTT, s (SD) & $25.15(3.136)$ & $25.48(4.131)$ & 0.327 \\
\hline $\mathrm{TT}, \mathrm{s}(\mathrm{SD})$ & $17.69(1.730)$ & $17.40(1.736)$ & 0.052 \\
\hline \multicolumn{4}{|c|}{$\begin{array}{l}n \text { number, } y \text { years, } B M / \text { body mass index, } S D \text { standard deviation, } V T E \text { venous thrombus embolism, } T G \\
\text { triglycerides, } T C \text { total cholesterol, } H D L-C \text { high density lipoprotein cholesterol, } L D L-C \text { low density } \\
\text { lipoprotein cholesterol, apo } A 1 \text { apolipoprotein } A 1, a p o B \text { apolipoprotein, } F I B \text { fibrinogen, } P T \\
\text { prothrombin time, } A P T T \text { activated partial thromboplastin time, } T T \text { thrombin time }\end{array}$} \\
\hline \multicolumn{4}{|l|}{ a: Pearson Chi-square test } \\
\hline b. Continuous correction test & & & \\
\hline
\end{tabular}

\section{Comparison of EA exponent}

In Table 2, the analysis of EA exponent showed significant differences in all the patients $(p=0.006)$, especially in female patients after stratifying by gender $(p=0.02)$. In males, there existed no differences in EA exponent between two groups $(p>0.05)$. Also, it could be noted that low EA exponent was a significant risk factor for postoperative DVT in both all the patients $(\mathrm{OR}=0.257,95 \% \mathrm{Cl}=0.110-0.599, \mathrm{p}$ $=0.002)$ and female patients $(\mathrm{OR}=0.205,95 \% \mathrm{Cl}=0.080-0.527, \mathrm{p}=0.001)$ when stratifying by gender after adjustment for age, $\mathrm{BMI}$, coagulation parameters, and all the other confounding factors in the multivariate analysis. 
Table 2

comparisons of EA exponent

\begin{tabular}{|c|c|c|c|c|c|c|}
\hline EA exponent & All & value & Females & $\begin{array}{l}p \\
\text { value }\end{array}$ & Males & $\begin{array}{l}p \\
\text { value }\end{array}$ \\
\hline \multicolumn{7}{|l|}{ Mean (SD) } \\
\hline DVT patients & $2.01(0.255)$ & \multirow[t]{2}{*}{0.006} & $2.00(0.265)$ & \multirow[t]{2}{*}{0.002} & $2.07(0.177)$ & \multirow[t]{2}{*}{0.407} \\
\hline $\begin{array}{l}\text { Non-DVT } \\
\text { patients }\end{array}$ & $2.09(0.386)$ & & $2.07(0.241)$ & & $2.18(0.697)$ & \\
\hline \multirow{2}{*}{$\begin{array}{l}\text { Multivariate } \\
\text { analysis }\end{array}$} & $\mathrm{OR}^{\mathrm{a}}(95 \% \mathrm{Cl})$ & \multirow[t]{2}{*}{0.002} & $\mathrm{OR}^{\mathrm{b}}(95 \% \mathrm{Cl})$ & \multirow[t]{2}{*}{0.001} & $\mathrm{OR}^{\mathrm{b}}(95 \% \mathrm{Cl})$ & \multirow[t]{2}{*}{0.233} \\
\hline & $\begin{array}{l}0.257(0.110- \\
0.599)\end{array}$ & & $\begin{array}{l}0.205(0.080- \\
0.527)\end{array}$ & & $\begin{array}{l}0.187(0.012- \\
2.936)\end{array}$ & \\
\hline \multicolumn{7}{|c|}{$E A$ erythrocyte aggregation, $S D$ standard deviation, $D V T$ deep vein thrombosis } \\
\hline \multicolumn{7}{|c|}{$\begin{array}{l}\text { a: Odds ratio adjusted for matching factors of gender, age, } \mathrm{BMI} \text {, and all the confounding factors in this } \\
\text { study }\end{array}$} \\
\hline
\end{tabular}

\section{ROC analysis of EA exponent}

In the ROC analysis, the AUC of EA exponent was 0.589 in all the patients $(95 \% \mathrm{Cl}=0.541-0.637, \mathrm{p}=$ 0.0003 ) and 0.593 in female patients $(95 \% \mathrm{Cl}=0.541-0.645 ; \mathrm{p}=0.0006)$ (Fig. 1).

\section{Discussion}

Accordingly, for patients who had primary KOA, our study detailedly assessed the potential association of preoperative EA exponent and postoperative acute DVT risk following TKA with routine anticoagulation of $\mathrm{LMWH}$. We found a tight correlation between the lower EA exponent and the increased risk of DVT, especially in female KOA patients. This emerging evidence appeared to supported an increasingly larger role of EA in the etiopathogenesis of DVT, which can be considered as a novel finding.

Actually, VTE is initiated by inflammation and blood stasis which may lead to the generation of thrombi rich in fibrin and erythrocytes[12]. From the perspective of hemorheology, erythrocytes can contribute to VTE under certain conditions mainly by promoting a rise in hematocrit, blood viscosity (BV)), marginate platelets and fibrin deposition, an elevation in EA, a decrease in erythrocyte deformability and/or its selfgenerated complex effects on blood clot structure and stability[12-14]. It can be indicated that erythrocytes are not only major components of venous thrombi and critical influence factors of VTE risk, but also actively incorporated into VTE pathophysiology[12]. Biologically, EA is commonly manifested by roleaux (linear arrays of stacked cells) or two- and three-dimensional aggregates with blood stasis in a 
osmotic gradient mechanism, which is thought to be reversible and shear dependent[18, 19]. The aggregates of erythrocytes can potentially increase BV and hydrodynamic resistance of larger vessels exactly as lower extremity veins $[15,19]$. Also, the extent of EA is the main determinant of low shear BV which is closely relevant to DVT[15, 19]. Accordingly, the underlying effects exerted by alterations of EA due to qualitative, quantitative, and interactive changes of erythrocytes in the pathogenesis of DVT is of considerable importance[11, 14].

In clinic, it is notable that the results seem to be somewhat inconsistent from the conclusions of the available studies[20-23]. Different confounding factors used for adjustment, testing time points for EA, and study populations can partly be the explanations. It has been expounded that the damage to the vessel wall, blood hypercoagulable state after surgery, and the venous stasis because of long-term bed rest are thought to be the 3 major reasons for the formation of postoperative DVT[24].

Considering our conclusions, it is tempting to speculate that the prothrombotic effects of erythrocytes in patients with a low EA exponent before surgery may be more prone to be affected by multiple factors during TKA surgery, which in turn lead to a relatively large increase of EA that ultimately have an imperceptible impact on DVT.

In spite of this, systemic hemorheological alterations may be not comparable to those in local areas where minimum disturbances can be more associated with DVT[11, 15]. To our best knowledge, the exact mechanisms linking EA and DVT await clarification and the results of our study may open new perspectives in this field.

A local hemorheological variation may played a crucial role in DVT formation and confirming the pathogenic significance of locally altered blood rheology in the development of DVT is much-required.

Noteworthily, both $\mathrm{OA}$ and obesity have been gradually recognized as lowgrade inflammatory disorders accompanied with an elevation of systemic inflammatory cytokines[25, 26]. Notably, there is mounting physiological evidence for the important role of inflammatory biomarkers in thrombogenesis[27, 28]. KOA is more common in obese people and these abnormally expressed inflammatory mediators in KOA patients may also affect EA in pleiotropic pathways. In this regard, the interactions between inflammatory state and EA have not been unequivocally established, further research is required before regulating EA can become therapeutic alternatives for DVT prophylaxis and treatment.

For the specific population of KOA patients who needed to undergo TKA, we firstly and systematically analyzed the correlation between preoperative EA exponent and post-surgery DVT risk. After adjustment for important confounders and effect modifiers, the findings emphasized the importance of EA in DVT prevention and are of a certain accuracy, which may have great guiding significance for follow-up clinical work. Still, the small sample size of males and the possible uncontrollable factors during TKA surgery may limit our results.

\section{Conclusions}


In conclusion, in primary KOA patients treated with TKA, preoperative EA exponent is independently associated with postoperative DVT. The result may be valuable for recalling the role of EA in thrombosis formation and optimizing prophylaxis methods.

\section{Abbreviations}

KOA: knee osteoarthritis; OA: osteoarthritis; DVT: deep vein thrombosis; VTE: venous thromboembolism; BMI: body mass index; TKA: total knee arthroplasty; TG: triglycerides; TC: total cholesterol; HDL-C: high density lipoprotein cholesterol; LDL-C: low density lipoprotein cholesterol; apo A1: apolipoprotein A1; apo B: apolipoprotein; FIB: fibrinogen; PT: prothrombin time; APTT: activated partial thromboplastin time; TT: thrombin time; EA: erythrocyte aggregation; OR: odds ratio; Cl: confidence interval; SD, standard deviation.

\section{Declarations}

\section{Acknowledgements}

We sincerely appreciate the assistance and patience from the nurses, the clinical laboratory, and the ultrasound department of our hospital.

\section{Authors' contributions}

$D X Y, Y P$, and $D$ WG designed the study and wrote the article. D XY, Y P, H ZH, and D WG collected the blood samples and provided the funding. D WG, X P, Z ND, and $L H$ did the operations. $D X Y, Y P$, and $X P$ did the data analysis. $Z$ ND made the postoperative DVT diagnosis. D XY, Y P, H ZH and D WG revised the manuscript. All authors read and approved the final manuscript.

\section{Funding}

The study was funded by the Basic Research Project of Changzhou science and Technology Bureau (CJ20200112) and the Youth Project "Science and Education" of Suzhou (KJXW2020068).

\section{Availability of data and materials}

The datasets used and/or analysed during the current study are available from the corresponding author on reasonable request.

\section{Ethics approval and consent to participate}

This study was conducted in accordance with the Declaration of Helsinki and approved by the ethical committee of The Third Affiliated Hospital of Soochow University. Informed consents were obtained from all the patients.

\section{Consent for publication}


Not applicable.

\section{Competing interests}

The authors declare that they have no competing interests.

\section{Author details}

${ }^{1}$ Department of Orthopedic Surgery, The Third Affiliated Hospital of Soochow University, Changzhou 213000, China.

${ }^{2}$ Department of Orthopedic Surgery, Changshu Hospital Affiliated to Nanjing University of Chinese Medicine, Huanghe Road 6, Changshu 215500, Jiangsu, China.

\section{References}

1. Lee H, Zhao X, Son YO, Yang S: Therapeutic Single Compounds for Osteoarthritis Treatment. Pharmaceuticals (Basel) 2021, 14(2).

2. Katz JN, Arant KR, Loeser RF: Diagnosis and Treatment of Hip and Knee Osteoarthritis: A Review. JAMA 2021, 325(6):568-578.

3. McClurg O, Tinson R, Troeberg L: Targeting Cartilage Degradation in Osteoarthritis. Pharmaceuticals (Basel) 2021, 14(2).

4. Block JA, Cherny D: Management of Knee Osteoarthritis: What Internists Need to Know. Med Clin North Am 2021, 105(2):367-385.

5. Hunter DJ, Bierma-Zeinstra S: Osteoarthritis. Lancet 2019, 393(10182):1745-1759.

6. Dai X, Ding W, Li H, Xu P, Huang Z, Zhu W, Liu J: Associations of Serum Lipids and Deep Venous Thrombosis Risk After Total Knee Arthroplasty in Patients With Primary Knee Osteoarthritis. Int J Low Extrem Wounds 2020, 19(1):51-56.

7. Zhang H, Mao P, Wang C, Chen D, Xu Z, Shi D, Dai J, Yao Y, Jiang Q: Incidence and risk factors of deep vein thrombosis (DVT) after total hip or knee arthroplasty: a retrospective study with routinely applied venography. Blood Coagul Fibrinolysis 2017, 28(2):126-133.

8. Geerts WH, Pineo GF, Heit JA, Bergqvist D, Lassen MR, Colwell CW, Ray JG: Prevention of venous thromboembolism: the Seventh ACCP Conference on Antithrombotic and Thrombolytic Therapy. Chest 2004, 126(3 Suppl):338S-400S.

9. Bulger CM, Jacobs C, Patel NH: Epidemiology of acute deep vein thrombosis. Tech Vasc Interv Radiol 2004, 7(2):50-54.

10. Prandoni P, Bilora F, Marchiori A, Bernardi E, Petrobelli F, Lensing AW, Prins MH, Girolami A: An association between atherosclerosis and venous thrombosis. N Engl J Med 2003, 348(15):14351441. 
11. Vaya A, Suescun M: Hemorheological parameters as independent predictors of venous thromboembolism. Clin Hemorheol Microcirc 2013, 53(1-2):131-141.

12. Aleman MM, Walton BL, Byrnes JR, Wolberg AS: Fibrinogen and red blood cells in venous thrombosis. Thrombosis research 2014, 133 Suppl 1:S38-40.

13. Litvinov RI, Weisel JW: Role of red blood cells in haemostasis and thrombosis. ISBT science series 2017, 12(1):176-183.

14. Weisel JW, Litvinov RI: Red blood cells: the forgotten player in hemostasis and thrombosis. Journal of thrombosis and haemostasis: JTH2019, 17(2):271-282.

15. Yu FT, Armstrong JK, Tripette J, Meiselman HJ, Cloutier G: A local increase in red blood cell aggregation can trigger deep vein thrombosis: evidence based on quantitative cellular ultrasound imaging. Journal of thrombosis and haemostasis: JTH2011, 9(3):481-488.

16. Xiaoyu D, Kai C, Zhihui H, Huan L, Naidong Z, Wenge D: Predictive value of preoperative erythrocyte electrophoresis exponent for acute deep vein thrombosis after total knee arthroplasty in patients with knee osteoarthritis. J Orthop Surg Res 2020, 15(1):496.

17. Rabinov K, Paulin S: Roentgen diagnosis of venous thrombosis in the leg. Arch Surg 1972, 104(2):134-144.

18. Baumler H, Neu B, Donath E, Kiesewetter H: Basic phenomena of red blood cell rouleaux formation. Biorheology 1999, 36(5-6):439-442.

19. Baskurt OK, Meiselman HJ: Erythrocyte aggregation: basic aspects and clinical importance. Clinical hemorheology and microcirculation 2013, 53(1-2):23-37.

20. Vaya A, Falco C, Simo M, Ferrando F, Mira Y, Todoli J, Espana F, Corella D: Influence of lipids and obesity on haemorheological parameters in patients with deep vein thrombosis. Thrombosis and haemostasis 2007, 98(3):621-626.

21. Alt $E$, Banyai $S$, Banyai $M$, Koppensteiner R: Blood rheology in deep venous thrombosis-relation to persistent and transient risk factors. Thrombosis research 2002, 107(3-4):101-107.

22. Vaya A, Mira Y, Ferrando F, Corella D, Aznar J: Does obesity constitute a risk factor for upperextremity deep vein thrombosis? Thrombosis and haemostasis 2005, 94(1):228-230.

23. Mira Y, Vaya A, Martinez M, Villa P, Santaolaria ML, Ferrando F, Aznar J: Hemorheological alterations and hypercoagulable state in deep vein thrombosis. Clinical hemorheology and microcirculation 1998, 19(4):265-270.

24. Saha P, Humphries J, Modarai B, Mattock K, Waltham M, Evans CE, Ahmad A, Patel AS, Premaratne S, Lyons OT et al: Leukocytes and the natural history of deep vein thrombosis: current concepts and future directions. Arteriosclerosis, thrombosis, and vascular biology 2011, 31(3):506-512.

25. Zhuo Q, Yang W, Chen J, Wang Y: Metabolic syndrome meets osteoarthritis. Nature reviews Rheumatology 2012, 8(12):729-737.

26. Blokhin IO, Lentz SR: Mechanisms of thrombosis in obesity. Curr Opin Hematol 2013, 20(5):437444. 
27. Rodriguez AL, Wojcik BM, Wrobleski SK, Myers DD, Jr., Wakefield TW, Diaz JA: Statins, inflammation and deep vein thrombosis: a systematic review. Journal of thrombosis and thrombolysis 2012, 33(4):371-382.

28. Niki Y, Matsumoto H, Hakozaki A, Mochizuki T, Momohara S: Rheumatoid arthritis: a risk factor for deep venous thrombosis after total knee arthroplasty? Comparative study with osteoarthritis. Journal of orthopaedic science: official journal of the Japanese Orthopaedic Association 2010, 15(1):57-63.

Figures

A

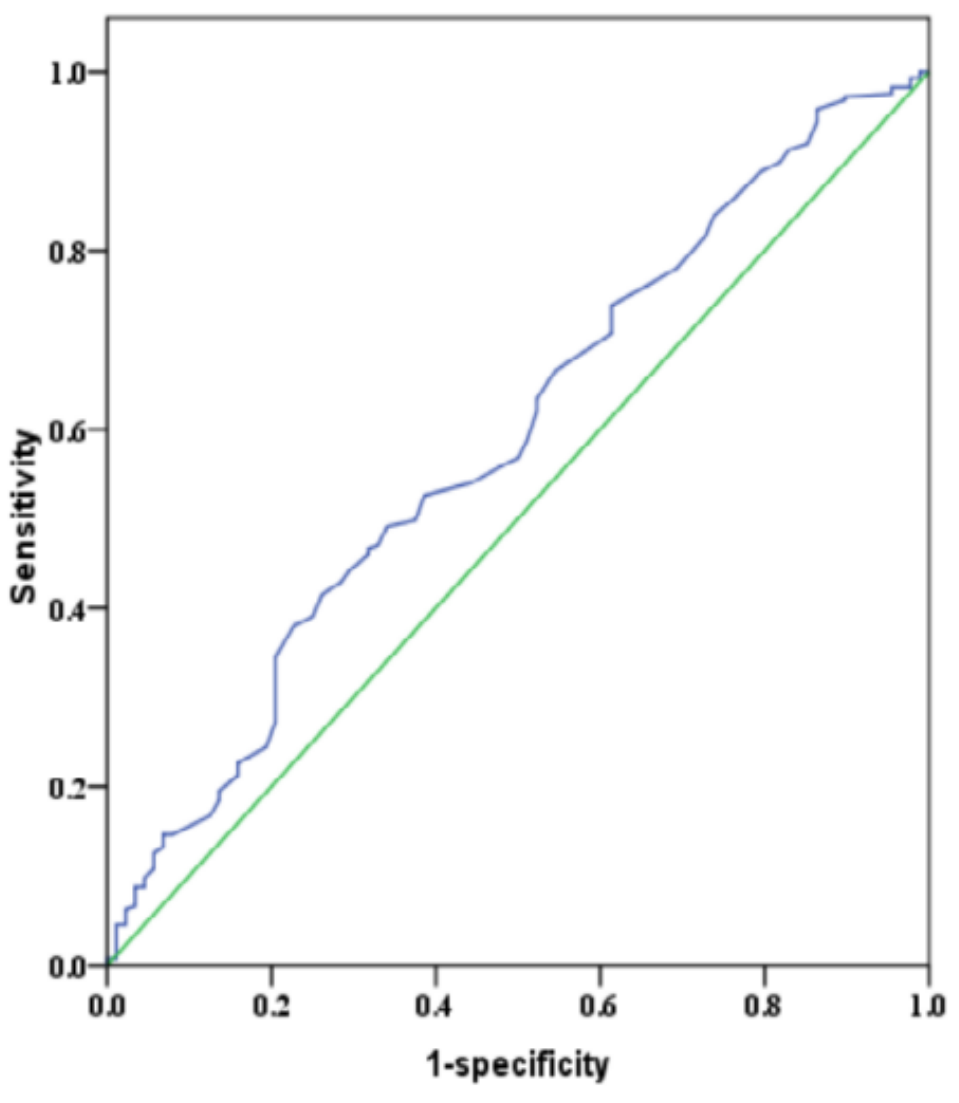

B ROC curve

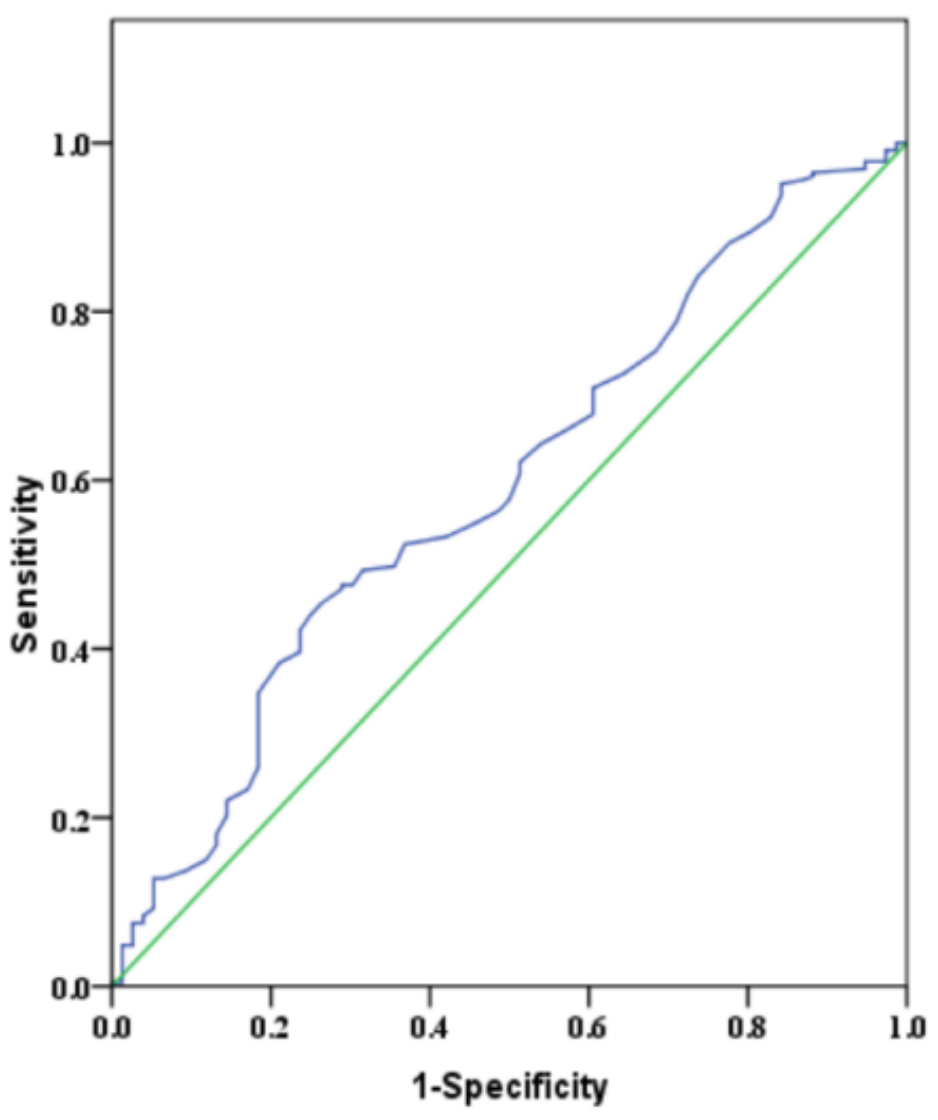

Figure 1

ROC analysis of EA exponent to predict postoperative DVT in all patients (A) and female patients (B). 